\title{
A breathing movement sensor for chest radiography during inspiration in children aged less than 3 years: a prospective randomized controlled study
}

\author{
Mari Honda ${ }^{1,}$, Rei Haruyama ${ }^{1, \S}$, Yasuo Sugiura ${ }^{1,2, *}$, Kaori Ohara ${ }^{1}$, Kazuya Mochigi ${ }^{3}$, \\ Yuzuru Kono ${ }^{1,3}$, Hiroyuki Shichino ${ }^{4}$, Hideko Uryu ${ }^{4}$, Tetsuya Mizoue ${ }^{5}$, Terumi Marutani ${ }^{6}$, \\ Takashi Ebihara ${ }^{6}$, Fumiya Uchiyama ${ }^{7}$, Satoshi Makise ${ }^{7}$, Hidechika Akashi ${ }^{1}$ \\ ${ }^{1}$ Bureau of International Health Cooperation, National Center for Global Health and Medicine, Tokyo, Japan; \\ ${ }^{2}$ International Health Care Center, National Center for Global Health and Medicine, Tokyo, Japan; \\ ${ }^{3}$ Department of Radiological Physics and Technology, National Center for Global Health and Medicine, Tokyo, Japan; \\ ${ }^{4}$ Department of Pediatrics, National Center for Global Health and Medicine, Tokyo, Japan; \\ ${ }^{5}$ Department of Epidemiology and Prevention, Center for Clinical Sciences, National Center for Global Health and Medicine, Tokyo, Japan; \\ ${ }^{6}$ Department of Clinical Trial Management, Center for Clinical Sciences, National Center for Global Health and Medicine, Tokyo, Japan; \\ ${ }^{7}$ Department of Radiology, National Center for Global Health and Medicine, Tokyo, Japan.
}

SUMMARY Chest radiographs should be obtained at the peak of inspiration so that radiological findings can be precisely interpreted. However, this is not easily achieved, particularly in young children who do not follow the instruction to hold their breath. We developed a sensor that detects the breathing movements and conducted a randomized controlled study to determine whether the sensor would increase the proportion of chest radiographs obtained in the inspiration phase. We recruited 124 infants and children aged less than 3 years, who visited the pediatric department of a general hospital in Tokyo, Japan, and allocated them into one of two groups: with-sensor and without-sensor groups. Overall, $81 \%$ of all images were obtained during inspiration. The proportion of chest radiographs taken during inspiration was not statistically different between the two groups ( $81 \%$ vs. $82 \%)$. In the with-sensor group, radiologic technologists were able to obtain chest radiographs of the same quality while not observing the chest movement, but the sensor. The use of the sensor did not increase the proportion of chest radiographs taken in the inspiration phase in this study. However, this null result may indicate the possibility of utilizing the sensor for automatizing chest radiography in the future.

Keywords chest radiographs, device, respiratory

\section{Introduction}

Since Wilhelm Conrad Röntgen discovered the X-rays in 1859 , chest radiographs have been one of the most commonly used diagnostic tools in medicine, both in adults and children (1). A chest radiograph produces images of the body structures in that area, such as the airways, lungs, heart, blood vessels, diaphragm, and the bones of the spine and chest (2), and provides support for the diagnosis of various diseases, including pneumonia, lung and mediastinal tumors, and heart failure.

In order to interpret chest radiographs, standardized definitions of radiological findings are required as well as appropriate training to utilize the definitions (3). However, the quality of radiographic images is crucial to precisely interpret the radiological findings. The quality of chest radiographs is determined by several factors, such as deep inspiration, proper positioning, inclusion of all areas of interest, and absence of blurring or external objects overlying the lungs. "Performed at peak of inspiration, except for suspected foreign body aspiration" is one of the quality criteria for proper diagnosis in children (4). In adults, 10 posterior ribs should be visible above the diaphragm when chest radiographs are obtained at peak of inspiration, according to the World Health Organization manual of diagnostic imaging (5). The criteria for young children and infants are different because of their smaller body size and different body composition. The optimal inspiration depth is demonstrated by eight visible posterior ribs in children younger than 3 years and nine posterior ribs in children between 3 and 7 years old ( 6 ).

To take appropriate chest radiographs in the 

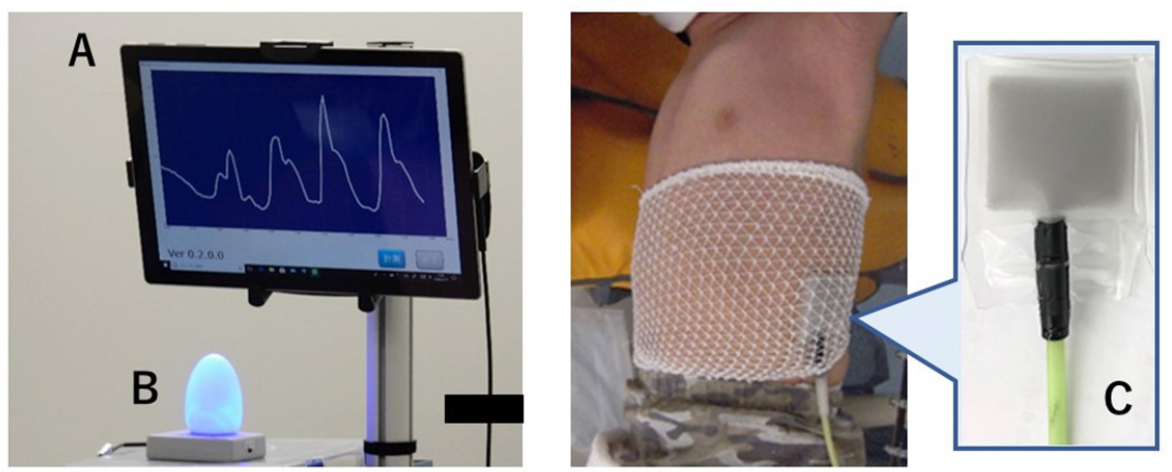

Figure 1. Images of the sensor device. (A) A monitor that shows the breathing waveforms; (B) An egg-shaped lamp indicating the inspiration phase; (C) An air pad detecting subtle body movement.

inspiration phase, patients are asked to stay still, take a deep breath, and then hold it for a few seconds. However, young children and infants are usually not able to hold their breath on command. Therefore, radiologic technologists judge the timing of the peak of inspiration by visually observing their thoracic movements. The high respiratory rates in children make it difficult to determine the appropriate timing. Furthermore, they usually do not cooperate and act up under unfamiliar circumstances. Few studies have analyzed whether chest radiographs were taken in the inspiration or the expiration phase. Tschauner et al. reported that only $49 \%$ of pediatric chest radiographs were obtained at the peak of inspiration (o).

Recent efforts to address this problem include training radiologic technologists and using assisting devices. Langen et al. developed a training program for radiologic technologists using video clips on how to obtain an infant's chest radiographs at the time of the deepest inspiration (7). After the simulation training with three infants in the video clips, radiologic technologists were more successful in performing chest radiography at the peak of inspiration, although the degree of improvement varied among individuals. Various types of body immobilization devices are also commonly used to reduce body movement and keep the individual in an appropriate position (8). However, to the best of our knowledge, there is no device that assists radiologic technologists to judge the timing of the peak of inspiration.

We developed and evaluated a sensor that detects the breathing movements in infants and young children aged less than 3 years. We hypothesized that the use of this sensor would increase the proportion of pediatric chest radiographs obtained during the inspiration phase.

\section{Materials and Methods}

\subsection{Development of the breathing movement sensor}

The original sensor was designed by THE YOSHIDA
DENTAL MFG. CO., LTD. (Tokyo, Japan) based on the patent JP, 5607912, B, and was used to measure vital signs, such as heart rate and breathing rate. For the purpose of our study, the sensor was modified to focus on the breathing condition. It consists of an air $\operatorname{pad}(5.5 \mathrm{~cm} \times 4 \mathrm{~cm} \times 0.56 \mathrm{~cm})$ that detects subtle body movement by the change in air pressure, a monitor that shows breathing waveforms (i.e., ascending waves during inspiration and descending waves during expiration), and an egg-shaped lamp that lights in green and beeps at the peak of inspiration (Figure 1A, 1B). The air pad was not completely radiotransparent. Therefore, to avoid interference with the chest image, the air pad was placed on the left lateral abdomen, which moves to pull down the diaphragm for breathing, and covered by an elastic mesh bandage $\left(\right.$ Presnet $^{\circledR}$ ALCARE Co., Ltd. Tokyo, Japan) (Figure 1C). We confirmed that the breathing waveforms produced by the air pad were consistent with those produced by CHESTAC-8900 (CHEST M.I., INC. Tokyo, Japan) in an adult test case, simultaneously using the air pad and the spirometer.

\subsection{Randomized controlled study}

We evaluated the effectiveness of this sensor by conducting a randomized controlled study to compare the proportion of chest radiographs obtained during the inspiration phase, with and without the sensor, among infants and young children aged less than 3 years. We conducted a pilot study of 10 patients to evaluate the feasibility before the main study.

\subsubsection{Study population}

We recruited children aged less than 3 years who visited the pediatric outpatient department during the regular working hours and were indicated to undergo chest radiography for clinical reasons by pediatricians at the Center Hospital of the National Center for Global Health and Medicine (NCGM), Tokyo, Japan, between 
September 2018 and October 2019. Children with lifethreatening conditions, severe chest wall malformation, and foreign body aspiration were excluded.

\subsubsection{Sample size calculation}

To identify the sample size for this study, we retrospectively assessed the pediatric chest radiographs taken at the hospital in May 2017. We found that 18 of the 59 radiographs (31\%) were not taken during the inspiration phase (i.e. eight posterior ribs were visible above the diaphragm). We expected that by using the sensor this percentage would be reduced to $20 \%$. With an $80 \%$ power, at the $5 \%$ significance level, the estimated sample size was calculated to be 124 (62 per group).

\subsubsection{Randomization}

We randomized participants by restricted randomization using random permuted blocks (9). The block size was two for 1:1 randomization to the intervention and control groups from numbers 1 to 124 . We allocated 62 "intervention" cards and 62 "control" cards in 124 nontransparent envelopes according to the randomization results. The envelopes were sealed and numbered from 1 to 124, and each contained a card showing either "intervention" or "control". These envelopes were saved at the radiology department and were not opened until the participants arrived at the department. When a participant would come to the department for imaging ordered by a pediatrician, a radiologic technologist opened one envelope in sequential order. The technologist used the sensor when there was an "intervention" card in the envelope. There was no crossover between the pediatricians who ordered the chest radiographs, researchers who conducted the randomization assignment, and radiologic technologists who opened the envelopes.

\subsubsection{Chest radiography}

Chest radiography was performed by certified radiologic technologists at the radiology department of the NCGM. Since the department operates in shifts, every technologist in the department was involved in the study. Children were separated from their caregivers in the radiographing room. The technologist decided to obtain chest radiographs in the posterioranterior or supine anterior-posterior position based on the child's age and condition. Immobilization devices were mostly used when radiographs were taken in the posterior-anterior position. After taking the radiograph, technologists recorded which modality they used to judge the imaging timing (i.e. breathing movement, waveform, light, sound, or a combination of these). One or two researchers attended almost all imaging procedures to observe the whole procedure.

\subsubsection{Interpretation of chest radiographs}

In this study, the inspiration phase in children younger than 3 years was defined by the presence of eight posterior ribs visible above the diaphragm. The two trained radiologists, with more than three years of experience, were assigned for interpreting the chest radiographs.

After 124 participants had undergone chest radiography, the two radiologists at the NCGM independently interpreted the radiographs and identified whether they were taken in the inspiration phase. Any discordance between the two readers was resolved by discussion to reach an agreement.

\subsubsection{Statistical analysis}

Data analysis was conducted using Stata 14 (Stata corporation, College Station, TX). The chi-squared test and Fisher's exact test were used to assess the correlation between sensor use and radiography in the inspiration phase. Stratified analyses were conducted by age and presence of cry.

\subsection{Ethical approval}

This study was approved by the Ethical Committee of the National Center for Global Health and Medicine (approval ID: NCGM-G-002310-00). Written informed consent was obtained from the caregivers of all participants.

\section{Results and Discussion}

A total of 125 children were recruited between September 20, 2018, and October 8, 2019. One child was excluded due to inadequate informed consent. Thus, 124 children were included in the analysis.

Table 1 shows the general characteristics of the study participants. The average age was $15.3(0-32)$ months, with about half being 1 year old. Eightyone percent of the participants were crying during the imaging. There were 61 participants in the withoutsensor group and 63 in the with-sensor group.

Table 1. General characteristics of the study participants

\begin{tabular}{lccc}
\hline & Without sensor & With sensor & Total \\
\hline Age groups (y) & & & \\
$0-1$ & $21(34 \%)$ & $22(35 \%)$ & $43(35 \%)$ \\
$1-2$ & $29(48 \%)$ & $31(49 \%)$ & $60(48 \%)$ \\
$2-3$ & $11(18 \%)$ & $10(16 \%)$ & $21(17 \%)$ \\
Crying & & & \\
Yes & $51(84 \%)$ & $50(79 \%)$ & $101(81 \%)$ \\
No & $10(16 \%)$ & $13(11 \%)$ & $23(19 \%)$ \\
& 61 & 63 & 124 \\
\hline
\end{tabular}


Table 2. Results of chest radiographs with and without sensor

\begin{tabular}{lcccc}
\hline & Without sensor & With sensor & Total & $p$ \\
\hline & & & & $0.9^{*}$ \\
Expiration & $11(18 \%)$ & $12(19 \%)$ & $23(19 \%)$ & \\
Inspiration & $50(82 \%)$ & $51(81 \%)$ & $101(81 \%)$ & \\
& 61 & 63 & 124 & \\
\hline
\end{tabular}

"determined by chi-square test

The proportion of radiographs taken in the inspiration phase was similar between the intervention (withsensor) and control (without-sensor) groups ( $81 \%$ vs. $82 \%, p=0.9$; Table 2).

In the intervention group, the imaging timing was judged according to the waveform on the monitor in 31 cases $(49 \%)$, the light signal of the egg-shaped lamp in 5 cases (8\%), the chest movement of the study participants in 21 cases $(33 \%)$, by the combination of light and waveform or sound and waveform in 3 cases $(5 \%)$, and unknown in 3 cases (5\%). After excluding the 21 radiographs taken by looking at the chest movement and 3 unknown cases, $87 \%$ (34/39) were obtained in the inspiration phase that examined the sole effect of the sensor.

When we stratified by age, we found that the proportion of images taken in the inspiration phase was much smaller among children $2-3$ years old $(7 / 21,33 \%)$ than in those $0-1(37 / 43,86 \%)$ and $1-2$ years old $(57 / 60$, $95 \%$ ). However, there was no significant difference in the proportion of images taken in the inspiration phase between the intervention and control groups in all three age groups (Fisher's exact test: $p=0.7, p=1.0, p=$ 0.7 , respectively). When participants were not crying during imaging, only $61 \%$ (14/23) of the radiographs were obtained in the inspiration phase. However, the proportion of chest radiographs obtained in the inspiration phase was similar regardless of the sensor use (Table 3).

The quality of chest radiographs largely depends on the skills of technologists. This is particularly true for pediatric chest radiographs because the timing of the inspiration phase exclusively relies on the eyes of the technologist. To the best of our knowledge, this is the first attempt to develop and evaluate a sensor that detects the breathing movements of infants and young children aged less than 3 years to judge the imaging timing in the inspiration phase on chest radiography.

Contrary to our hypothesis, our study showed no statistical difference in the proportion of radiographs taken in the inspiration phase according to the sensor use. Moreover, the overall proportion of chest radiographs obtained in the inspiration phase in our study (81\%) was higher than that in our pilot study $(69 \%)$. This proportion is also higher than that reported in a previous study (49\%) (6). One of the reasons might be observer bias (10). One or two researchers attended
Table 3. Result comparison between non-crying and crying participants

\begin{tabular}{lrrrr}
\hline & Without sensor & With sensor & Total & $p$ \\
\hline $\begin{array}{l}\text { No cry } \\
\text { Expiration }\end{array}$ & $4(40 \%)$ & $5(38 \%)$ & $9(39 \%)$ & $1.0^{* *}$ \\
Inspiration & $6(60 \%)$ & $8(62 \%)$ & $14(61 \%)$ & \\
Cry & & & & $1.0^{* *}$ \\
Expiration & $7(14 \%)$ & $7(14 \%)$ & $14(14 \%)$ & \\
Inspiration & $44(86 \%)$ & $43(86 \%)$ & $87(86 \%)$ & \\
\hline
\end{tabular}

** determined by Fisher's exact test

almost all imaging procedures during the study to observe the procedure and this might have affected the technologists' behavior. Additionally, in our pilot study, we analyzed all chest radiographs, including those obtained in the emergency department during non-working hours, which may have had lower quality than those taken at the radiology department during regular working hours. At the emergency department in our hospital, there is less staff and no immobilization device for pediatric chest radiography. Without the device, radiologic technologists usually have to hold infants and young children to maintain their good position during the procedure, which may distract them from optimizing the imaging timing.

Although the proportion of chest radiographs obtained in the inspiration phase in the overall results was high, the participants who did not cry and those in the 2-3 years age group was relatively low. Radiologic technologists often had difficulty in detecting chest movement in non-crying children. Moreover, elder children might be difficult to keep in the required position during the procedure. The immobilization device in our hospital could be used for children with body weight up to $15 \mathrm{~kg}$, but some children in our study did not allow us to use it. Unfortunately, the sample size of non-crying participants and also those in the 2-3 years age group were both not sufficient to conduct sub-analyses in this study. In a future study, it might be intriguing to target non-crying children or recruit adults having difficulties communicating and/or following directions.

Among the 39 chest radiographs obtained by observing the waveform, light, or according to the beep sound of the sensor, 34 (87\%) were taken in the inspiration phase. This result indicates that this type of sensor could be utilized for automatizing chest radiography. Ideally, artificial intelligence could be used to analyze the patterns of a respiratory waveform to automatically shoot chest radiographs at the peak of inspiration. This new device may reduce the workload and occupational radiographic exposure of radiologic technologists without influencing the quality of images. Respiratory gating in computed tomography (CT), positron emission tomography/CT, or magnetic resonance imaging, in particular for radiation therapy, 
have been already put into practical use (11). Our sensor is simpler than those systems and could, therefore, lead to a better quality of chest radiographs in a cost effective way. Moreover, in response to economic growth and the changes in social development, there has been an increasing demand for diagnostic imaging in low- and middle-income countries. Although there is often a shortage of qualified radiologic technologists, this device could be of assistance in those countries.

The study has several limitations. First, doubleblinding was not feasible. The two radiologists who interpreted the chest radiographs were blinded to the participants' allocation; however, the radiologic technologists and participants recognized it because of the use of the sensor in the intervention group and non-use in the control group. We could have placed a nonfunctional air pad in the control group; however, the radiologic technologists would still not have been blinded. Second, the subgroup analysis by category or severity of the diseases was not conducted since the focus of our study was on the effect of the sensor regardless of disease or condition within our criteria. Third, our estimation of the proportion of chest radiographs obtained in the inspiration phase in the control group was lower than the actual result. Therefore, our sample size was too small to have sufficient statistical power. Finally, we could not make children stop crying during the procedure even though the crying affects the chance to obtain radiographs in the inspiration phase (12). In general, children cry during the radiographic examination because they feel stress, fear, or anxiety (13). For future research, it might be interesting to include adult participants who were not able to move and communicate with others.

In conclusion, in this study, the use of the body movement sensor did not increase the proportion of chest radiographs taken during the inspiration phase among infants and young children aged less than 3 years. However, this null result may indicate the possibility of utilizing the sensor for automatizing chest radiography in the future.

\section{Acknowledgements}

This research was supported by a grant from the National Center for Global Health and Medicine, Japan (19A2010). The sensor applied in the current research was provided by THE YOSHIDA DENTAL MFG. CO. LTD. (Tokyo, Japan) during the study period based on the research contract between the company and the National Center for Global Health and Medicine, Japan.

We thank all members of the Center Hospital of the National Center for Global Health and Medicine for their contribution to this study, as follows: Ayumi Mizukami, Junko Yamanaka, Keiji Goishi, Mizue Tanaka, Masao Kaneshige, Minsoo Lee-Yoshimoto, Mami Shimada, Satoshi Takasago, Tomohisa Akamatsu, Yuri
Suzuki, Yukari Atsumi, Tomomi Ota, Haruka Aihara, Mayu Koto, Chihiro Ajimi, Yuko Tsuchida, Haruka Yamanaka, Hideki Funazaki, Yoshinobu Yajima, Futoshi Matsunaga, Azusa Minagawa, Ken Matsumoto, Satomi Yamamoto, Takumi Iwase, Daisuke Yamazaki, Daisuke Ishibashi, Ayumi Suetaka, Yoshikazu Takahashi, Tomoko Oikawa, Nao Fujiki, Shun Otsuka, Keiichi Kurihara, Kenta Hashimoto, Kento Mitani, Kotaro Kijima, Megumi Fujii, Daisuke Muramatsu, Kazuki Shinagawa, Masashi Hayano, Tsuyoshi Sakai, Hiroyuki Takano, Tsubasa Sugihara, Misato Terashima, Masaki Nagai, and Toshihiko Hashizume.

Financial Disclosures: The authors declare no conflicts of interest associated with this manuscript.

Trial Registration: UMIN-Clinical Trial Registry; UMIN000031052; January 29, 2018.

\section{References}

1. Greene R. Radiologic history exhibit. Francis H. Williams, MD: Father of chest radiology in North America. Radio Graphics. 1991; 11:325-332.

2. Puddy E, Hill C. Interpretation of the chest radiograph. Continuing Education in Anaesthesia, Critical Care, \& Pain. 2007; 7:71-75.

3. Cherian T, Mulholland EK, Carlin JB, Ostensen H, Amin R, de Campo M, Greenberg D, Lagos R, Lucero M, Madhi SA, O'Brien KL, Obaro S, Steinhoff MC. Standardized interpretation of paediatric chest radiographs for the diagnosis of pneumonia in epidemiological studies. Bull World Health Organ. 2005; 83:353-359.

4. European Commission. European guidelines on quality criteria for diagnostic radiographic images in paediatrics. Luxembourg, 1996; pp. 27. https://www.sprmn.pt/pdf/ EuropeanGuidelinesEur 16261.pdf (accessed March 23, 2020).

5. Ostensen H, Pettersson H, eds. The WHO manual of diagnostic imaging. World Health Organization, 2003; chapter3, pp. 6.

6. Tschauner S, Marterer R, Gubitz M, Kalmar PI, Talakic E, Weissensteiner S, Sorantin E. European guidelines for AP/PA chest X-rays: routinely satisfiable in a paediatric radiology division? Eur Radiol. 2016; 26:495-505.

7. Langen HJ, Kohlhauser-Vollmuth C, Sengenberger C, Bielmeier J, Jocher R, Eschmann M. Performing chest $\mathrm{X}$-rays at inspiration in uncooperative children: the effect of exercises with a training program for radiology technicians. Radiol Res Pract. 2014;2014:312846.

8. Kohda E, Tsutsumi Y, Nagamoto M, Gomi T, Terada H, Kawawa Y, Masaki H, Shiraga N. Revisit image control for pediatric chest radiography. Radiat Med. 2007; 25:60-64.

9. Efird J. Blocked randomization with randomly selected block sizes. Int J Environ Res Public Health. 2011; 8:15-20.

10. Mahtani K, Spencer EA, Brassey J, Heneghan C. Catalogue of bias: observer bias. BMJ Evid Based Medicine. 2018; 23:23-24.

11. Frood R, McDermott G, Scarsbrook A. Respiratory-gated $\mathrm{PET} / \mathrm{CT}$ for pulmonary lesion characterisation-promises and problems. Br J Radiol. 2018; 91:20170640.

12. Frank ED, Long BW, Smith BJ. Merrill's atlas of 
radiographic positioning and procedures - E-Book. Elsevier Health Sciences, 2013; pp. 118.

13. Björkman B, Golsäter M, Enskär K. Children's anxiety, pain, and distress related to the perception of care while undergoing an acute radiographic examination. J Radiol Nurs. 2014; 33:69-78.

Received March 23, 2020; Revised April 7, 2020; Accepted May 11, 2020.
${ }^{\S}$ These authors contributed equally to this work.

*Address correspondence to:

Yasuo Sugiura, Bureau of International Health Cooperation, National Center for Global Health and Medicine, 1-21-1 Toyama, Shinjuku-ku, Tokyo 162-8655, Japan.

E-mail: yasugiura@it.ncgm.go.jp

Released online in J-STAGE as advance publication May 17, 2020. 